\title{
A low protein diet during early gestation in sheep detrimentally impacts hepatic glucose metabolism in the adult offspring
}

\author{
D. S. Gardner ${ }^{1}$, P. Rhodes ${ }^{1}$, A. Karamitri ${ }^{1}$, E. Glaab ${ }^{2}$ and S. M. Rhind ${ }^{3}$ \\ ${ }^{1}$ School of Veterinary Medicine and Science, University of Nottingham, LE12 5RD, UK, ${ }^{2}$ School of Computer Science, \\ University of Nottingham, NG8 1BB, UK and ${ }^{3}$ Macaulay Land Use Research Institute, Aberdeen AB15 8QH, UK
}

Differences in maternal diet can account for variation in the metabolic competence of the subsequent individual as an adult. 'Developmental programming' may impair fetal organ development leading to a limitation in function as an adult and/or increase the rate of agerelated organ decline for example under conditions of obesity. Here, we have tested the interaction between prenatal nutritional 'thrift' and postnatal nutritional excess on gluco-regulatory functions in an ovine model.

Seventy-four Scottish Blackface ewes were randomly assigned to receive either a control protein diet with adequate energy (18\% protein; CP, $n$ 20) or low protein diet (9\% protein) fed during early gestation (0-65d, term $\sim 147 \mathrm{~d}$; LPE, $n$ 37) or late gestation (65-147 d; LPL, $n$ 17). At $65 \mathrm{~d}$ a proportion of ewes was euthanised for fetal sampling. At term, remaining ewes lambed naturally, were weaned at 10 weeks and a random sample of offspring studied longitudinally when lean (1.5 years of age) and after 6 months exposure to an obesogenic environment. Body composition was determined by dual-energy absorptiometry and glucose and insulin tolerance tests were conducted with appropriate sampling intervals. At post mortem, muscle and hepatic tissues were sampled for expression and abundance of relevant gluco-regulatory genes.

The diets had little effect on maternal weight and body composition through gestation or on fetal weights at $65 \mathrm{~d}$. Term weight was reduced by $\sim 500 \mathrm{~g}(P=0.001)$ in LPL $v$. other groups but, by weaning, body weight was similar between groups and growth rate to adulthood was not different. Homeostasis model assessment of baseline glucose and insulin concentrations indicated relative insulin resistance in male LPE (Fig - left panel). Indeed, when challenged with a GTT, the incremental insulin AUC was significantly greater in male LPE when obese (Fig - centre panel) but not when lean (unpublished results). Molecular quantification of glucose-insulin pathways in muscle and liver indicated specific down-regulation of the hepatic insulin, but not lipid, pathways in male liver only (Hepatic Akt only shown for clarity; Fig - right panel). Muscle insulin-signalling pathways were unaffected as determined by microarray (Affymetrix, U133 chip; www.array-mining.net).
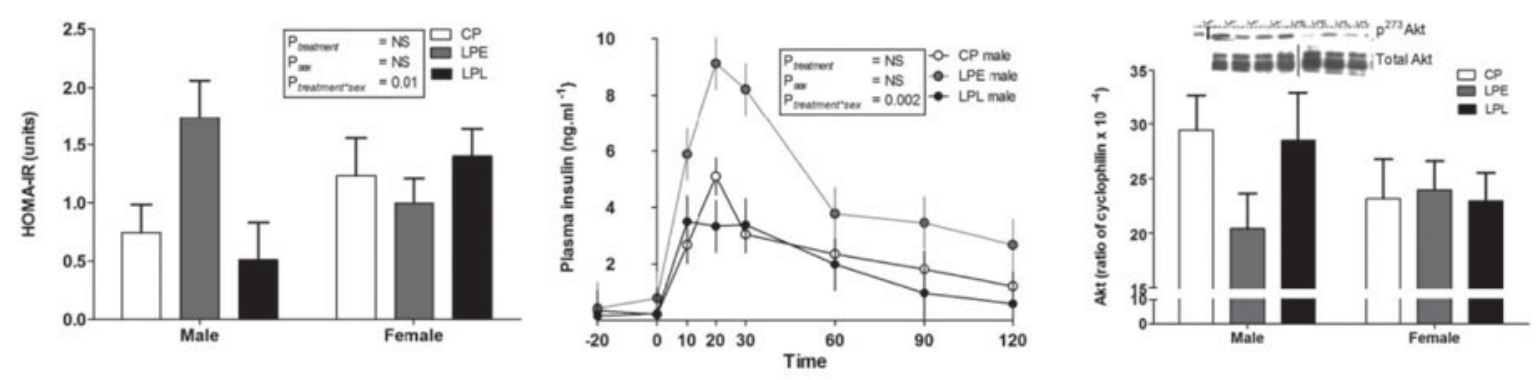

The data suggest that a maternal, low protein, diet during early gestation specifically impacts upon the function of the resulting adult liver, such that the offspring appear more susceptible to large excursions in plasma insulin during gluco-regulatory challenges. The insulin sensitivity of offspring muscle, the largest single source of insulin-stimulated glucose uptake, was largely unaffected. Thus, obesity appears to exacerbate any functional deficits inherent in low protein exposed offspring in sheep, but those offspring born of low birth weight were largely unaffected, illustrating that nutritional quality is far more important than nutritional quantity especially during sensitive developmental phases of growth. 